\title{
Assessment at Al-Ameer signalized intersection in Samawa city
}

\author{
Hasan Joni ${ }^{1}$, Mafaz Hikmatt ${ }^{1}$ \\ ${ }^{1}$ Building and Construction Engineering Department, University of Technology, Baghdad, Iraq
}

\begin{abstract}
The aim of this paper is to evaluate traffic performance of Al-Ameer signalized intersection in Samawa city, which fits with the prevailing conditions and geometric properties of the intersection. The technique of video recording has used for collection the traffic volume data for all approaches. These data are abstracted from video films. SYNCHRO 8 software was used to evaluate and analyze the intersection and choose the best suggestion. The evaluation process result showed that the intersection is operated with level of service (LOS F). By suggestion of several strategies which vary from signal optimization to geometric improvements. The best solution has been found by suggestion an overpass at the east-west direction, and the level of service has improved from (LOS F) to (LOS C), this result is considered an acceptable and economical solution for the existing problems at intersection.
\end{abstract}

\section{Introduction}

Most of governmental agencies require studies for traffic impact, also known as analyses of traffic impact and assessments of traffic impact, for the developments that produce a significant amount of new traffic or changing in existing traffic patterns.

The network of road transportation of any country plays an essential role in the economical development, then, ensure that there are road networks connecting all parts of country and that the transportation infrastructures is in good, the perfect physical conditions is very critical. Lacking in suitable transportation network reaching to viable parts of the country becomes a huge economical challenge [1].

The systems of transportation are the motor of the economical activities in whole urban societies the entire world, consequently sustaining a livelihood of a people living in. The standard facilities of urban transportation which contain roads, railways, airways and waterways. Through these, the major proportion is road network. Logically, most of researches and planning studies have concentrated on the road systems. In substance, system of road transportation is major factor in economic activities of the most urban areas. In current times, a large increase in traffic and transport demand have seen in many cities, which has thus, lead to decay in the capacity and inactive performance of traffic system [2].

\section{Intersection}

An intersection is the area where two or more roadways connect. It includes the roadway and roadside facilities available for traffic movement.

For many years, measurement of the performance level of signalized intersections has primarily focused on vehicle delay. Besides vehicle delay, there are another performance measurements like the spatial volume of queues, and the number of vehicle stops at intersection approaches have been found to play an important part in the performance of evaluation of signalized intersections in developing countries, where most of the times the intersections remain near or at oversaturated status. Hence, situation of go and stop is very frequent at the intersection. These measurements not only related to the level of service (LOS) that provided to the drivers, but, also to the air pollution and fuel consumption level which generated by the vehicles that crossing the signalized intersection. particularly, estimations of vehicle stop play an essential factor in determining consumption of vehicle fuel and emission at intersection approaches, estimation of queue length is significant not only for designing of lanes, but also to guarantee that the operations of traffic signal do not influence in queues which spill backing into the upstream intersection [3].

Signalized intersections could be defined are the basic elements in the system of urban transportation and carry the heavy traffic for motorized and non-motorized vehicles and pedestrian, which, create many conflicts between turning and crossing, merging of maneuvers. For a diversity of reason like economic, auto ownership growth and population, increasing of traffic demand could exceeding the capacity of the intersection through the peak hour periods. Consequently, deterioration of traffic conditions deteriorates and safety worsens. Congestion and dangerous of traffic condition lead to increase emission, accidents, consumption of fuel, and noise, thus, a quality of life, resources of world energy and global atmospheric conditions lead to deteriorate service [4].

An intersection is any site or place where many users mix and compete for time within the same area. Intersection takes many shapes and forms, range from complex junctions to driveways to the meeting of two ways. They are oftentimes defined by their operations and layout: traffic signals, T-junctions and roundabouts. 
Compactness, Simplicity, low speed and eye contact are favored in intersection design [5].

Performance of Intersection as calculated by the delay is an action of different factors include, turning movement demands, signal timing plan, traffic stream compositions, intersection geometry, pedestrians volume, temporary variation in traffic demand, driver characteristics, headway distributions at each traffic flow, conditions of road surface, visibility and weather. Some of these factors are constant such as (signal timing planning and geometry) while others differ like (traffic demand, weather, etc.) [6].

\section{Delay time types}

There are different types of delay time that could be calculated at intersections, and every one fills an alternate need to the transportation engineer. Intersection delay may include two components: stop (queue) delay and control delay. Stop delay, or queue delay, is difficult to quantify due to its stochastic nature that is affected by random arrival. The sophisticated techniques could work better in estimation of queue delay, but are often impractical for planning models due to this requirement of intense data. It is often hard to find a well-balanced queue delay model for integration into planning models. Estimation procedure of the signalized intersections capacity and LOS are worked around the idea of moderate control delay per vehicle. The control delay could be defined as a part of the total delay assigned to the operations of traffic signal for signalized intersections $[7,8]$. The control delay (overall delay) can be grouped into decelerations delay, accelerations delay and stopping delay:

a- Stopping delay is simpler to be measured, typically, a transportation experts define the stopping delay as the delay that happens when the vehicles are completely stopped or immobilized,

b- The delay that happens by an acceleration or deceleration of vehicle is categorized as acceleration and deceleration delay, respectively.

In HCM2000 (TRB 2000), the control delay is included in initial deceleration delay, queue move up time, stopping delay, and final acceleration delay, however, in prior versions it included just the stopping delay. Besides the control delay, there are other kinds of delay time which vehicles experience at the signalized intersections. This kind of delay is known as "geometric delay" which is the time lost because of the geometrical layouts of intersection. Geometric delays might be huge for the turning movement. So, the total delay of a vehicle is the whole sum of control delay plus to geometric delay [9].

\section{Al-Ameer signalized intersection as a case study}

This intersection in Samawa City is a four leg signalized intersection which is operates as a fixed time signal control and has four signal phases, and represents one of the most important intersections in the city center, where many lively activities happen at this intersection. The south approach suffers from high traffic volume due to many shopping centers, offices, cafes and others activities exist in this street and the west approach leads to neighborhoods and residential areas, and as shown in the Figure 1.

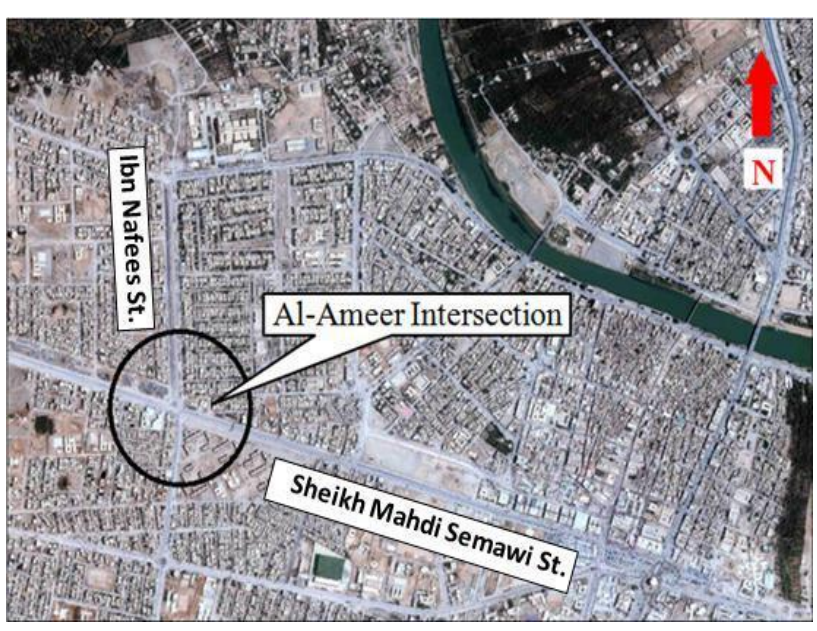

Fig. 1. The Study Area and the Selected Intersection

The layout diagram of this intersection is shown in Figure (2) as in the following.

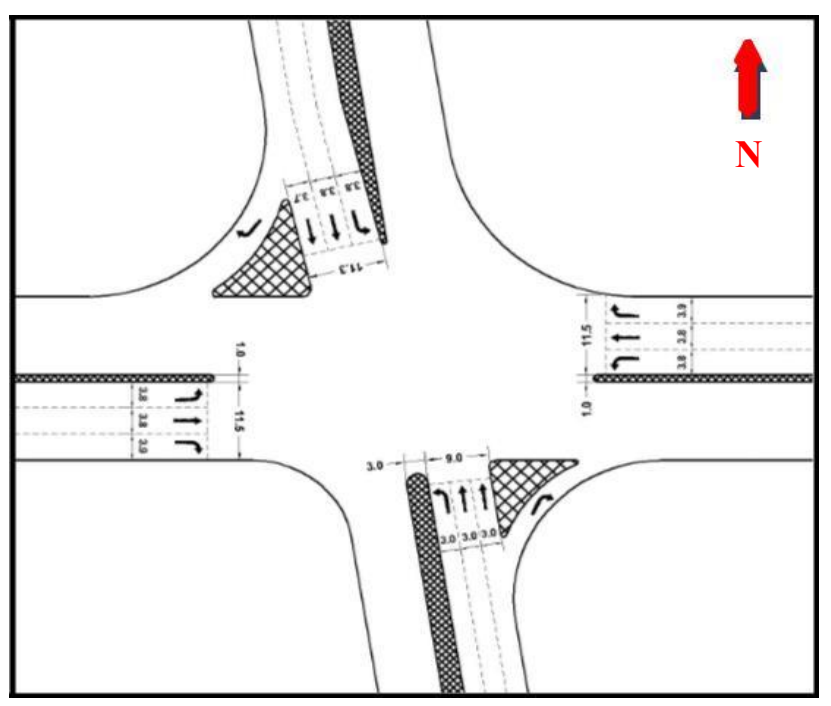

Fig. 2. The layout diagram of Al-Ameer Intersection

\section{Signal phasing and timing}

In Samawa City, to control the traffic, fixed time traffic signals are being used for a significant number of years. Unfortunately, rather than using any traffic engineering knowledge, these signals have been timed by traffic police from arbitrary judgment only. As a result, they became ineffective to serve the purpose properly and efficiently. Therefore, this research assumed that the selected intersection work actuated system by traffic police and four phase signals (each approach with one phase) and use the maximum actual green time.

\section{Major parameters for evaluating intersection operations}

Capacity, delay and level of service are the main parameters for evaluating the operations of intersections. 


\subsection{Capacity}

The Highway Capacity Manual (2000) demonstrates the capacity of facility as " the maximum hourly rate that a person or a vehicle can reasonably be expected to traverse a point or uniform section of a lane or roadway through a given time period under the prevailing roadway, traffic and control conditions " [10].

\subsection{Delay}

Delay is one of the most important factors that are used at the optimization procedure of traffic signal timing. Moreover, delay is the main parameter in calculating the level of service (LOS) providing to the drivers at signalized intersection. Delay, is a factor that is difficult to be estimated because it contains the delay related to the deceleration to the stopping, the stopping delay and the delay related to the acceleration from the stopping [11].

\subsection{Level of service}

Level of service (LOS) is defined in terms of the average total vehicles delay of the whole movements through an intersection. Vehicle delay is a way of evaluation many intangible parameters which include driver discomfort, loss of travel time and frustration. In particular, levels of service (LOS) criteria are expressed in terms of average delay per vehicle through a specific time period (such as, the AM peak hours). Vehicle delay is a complicated measurement based on several variables, include signal phasings (i.e., progress of movements during the intersection), length of signal cycle and volumes of traffic with respect to capacity of intersection [7].

\section{Methodology of data collection}

To fulfill the objectives of the study, it was important to go ahead with the designed procedures to gather the required data. These procedures are classified into four steps as explained below:

1. Describing the study area limitations.

2. Defining the peak hour periods.

3. Defining the specified input data for the selected software.

\section{Geometric data}

The main objective behind collecting the geometrical data for the signalized intersections is to evaluate and enhance the traffic through increasing the width of a lane, adding a new lane, creating an overpass or underpass.

Some of geometric data have been collected via using GIS techniques by using map measurements and depending on the available satellite images for Samawa city with precision of $60 \mathrm{~cm}$, and updated to 2013 . The slopes of all intersections are done by using the level instrument. Field measurements are obtained by using measuring tape to get such as, the median width and lane width, some of geometric features are obtained by observation such as; existence of exclusive LT or RT lanes, number of lanes per approach and Parking.

\section{Traffic volume data}

The traffic volume data are collected for two week by video recording technique for three different periods: morning peak period of (7:00- 9:00 A.M.), noon peak period of (1:00-3:00 P.M.) and evening peak hour of (5:00 -7:00 P.M.), after many personal observations, and pilot surveys have been done in the study area. Also, many personal interviews are done with the interested people such as traffic policemen at the study area and several road users in order that determining the peak hour periods for collecting the traffic data.

The video recording is made in normal conditions, good weather (dry), and good visibility, and by using a video camera SONY HANDYCAM lens $8 \mathrm{~mm}$, and later copied onto solid disk. From all of these considerations, it was concluded that Al-Ameer intersection has morning peak hour of (7:30 - 8:30) A.M. as shown in Figure 3.

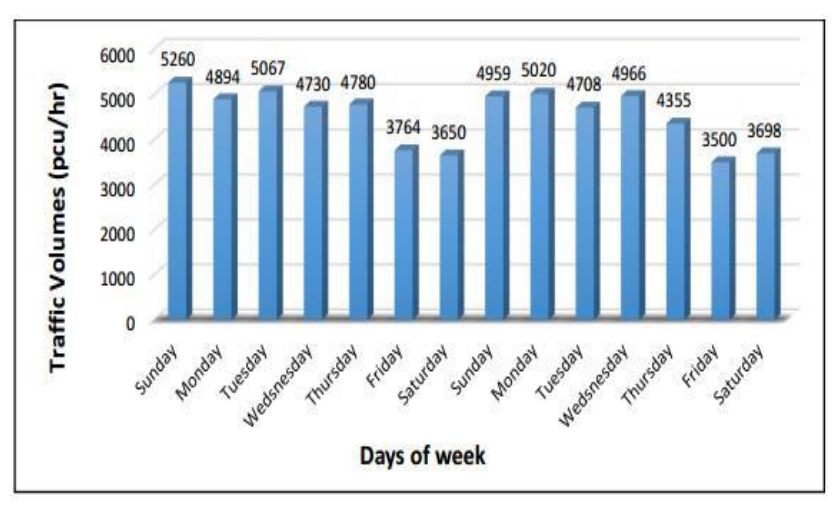

Fig. 3. Total Traffic Volume at Al-Ameer Intersection

\section{Traffic volume data for intersection}

This data include calculation of the traffic volumes abstracted from the video recording for each approach at intersection, also, composition of traffic (passenger car ,private car ,heavy vehicle, bus and motorcycle), and volumes of turning movement (though, left, right, and $\mathrm{U}$ turn). The traffic volume is transformed to passenger car units (PCU) by multiplying every vehicle kind by its factor according to HCM 2000.

\section{Traffic signal data}

For the signalized intersections the cycle length, phase length, green time and all red time are measured from the video film.

- Cycle length: is the whole (total) time for the signal to finish one cycle, and represented by second.

- Phase length: is a portion of the cycle length specified to any group of the traffic movements that receive the right of way together through one or more interval.

- Yellow time : is the value of time of the yellow interval , generally, the value of time must to be between 3 to 5 second, based on width of street, speed of approach and the local standards .The minimum value of time permitted by the SYNCHRO 8 is 2 second and the maximum is 10 second [12]. 
- All red time: is the value of time for all red intervals that follow the yellow intervals. All red time must be of a suitable interval to allow time at the intersections to clear before the traffic is released [12].

\section{Data abstraction}

Data abstraction was based on sessions of 15 minute periods of recorded data. The choice of a 15 minute period was depended on these considerations:

1. To guarantee that the sample is adequate to give meaningful results.

2. The traffic flow should be effectively constant during the period without incidents.

3. Decreasing of observer fatigue and subsequent mistakes. To get the required data from a video film, it was important to play back the film many times. To guarantee that the video film begins at the same point in time.

\section{Application of SYNCHRO 8 software}

SYNCHRO 8 was applied to simulate the existing traffic flow for the selected intersection. It is an entire programming package for modeling, optimization, and simulation traffic system. The results in Table 1 show that most the intersection suffers from an oversaturation condition with high total delay values and unacceptable level of service (LOS F).

Table 1. Performance Analysis for Al-Ameer Intersection.

\begin{tabular}{|c|c|c|c|c|}
\hline 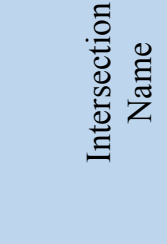 & 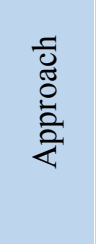 & 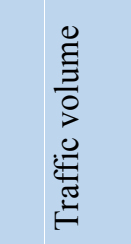 & ֻุ & 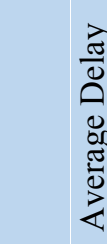 \\
\hline \multirow{4}{*}{ Al-Ameer } & North & 890 & $\mathrm{~F}$ & 84.0 \\
\hline & South & 1682 & $\mathrm{~F}$ & 286.0 \\
\hline & East & 1302 & $\mathrm{~F}$ & 154.1 \\
\hline & West & 1381 & $\mathrm{~F}$ & 184.5 \\
\hline \multicolumn{3}{|c|}{ Summary } & & \\
\hline \multicolumn{3}{|c|}{$\begin{array}{r}\text { Average Delay }=191.5 \mathrm{sec} \\
V / C=1.97\end{array}$} & \multicolumn{2}{|c|}{$\mathbf{L O S}=\mathbf{F}$} \\
\hline
\end{tabular}

\section{Improvement of intersection}

This section presents the improvement strategies applied to the traffic flow at the selected intersection, through the application of the calibrated software SYNCHRO 8.

This section divides the improvement strategies into two parts according to method and software applied. The first part could be named as, "the cycle length optimization", and include cycle length optimization, splits optimization, and phasing sequences. The second section could be name marking, pavement widening and construction an overpass.

\subsection{Cycle length optimization method}

The optimization of Traffic signal timing considered one of the most cost-effective methods to reduce the costs of vehicle operating and to improve traffic flow performance along urban streets. SYNCHRO 8 includes a number of optimization kinds. It optimizes the cycle lengths, phase sequences and split times in order to decrease the delay time and stop. These types are applied to the selected intersection in the same order and as shown in Figure 4. [13].

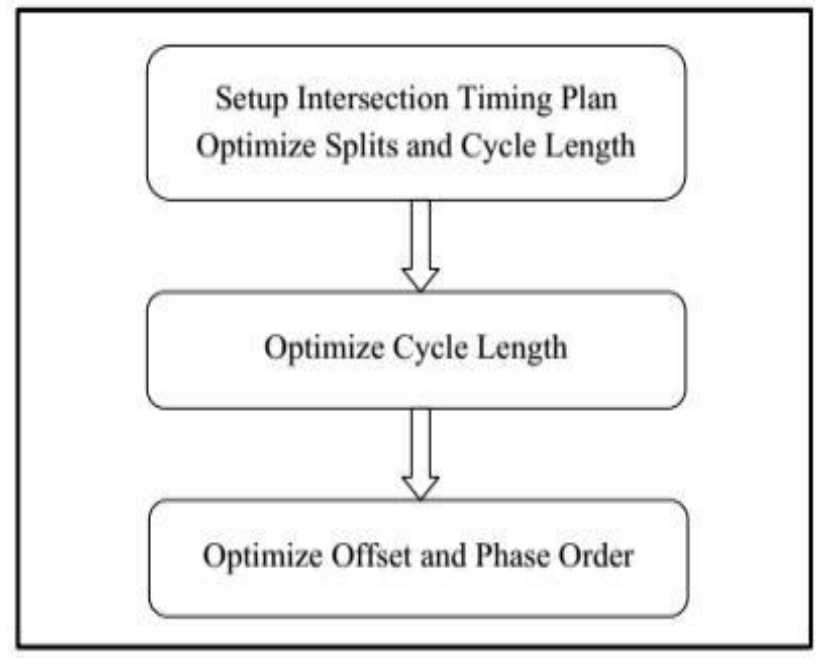

Fig. 4. Optimization Steps in SYNCHRO 8

SYNCHRO 8 begins with the shortest cycle length, and then optimizes the splits for that cycle length. If the split for every phase is not capable to clear out the percentile critical traffic, SYNCHRO 8 will have to try a higher cycle length till the percentile critical traffic is cleared.

The optimize splits order will automatically set the splits for all the phases. Time is splited based on every lane group, and the volume of traffic is divided by its adjusted saturation flow rate. Splits optimization will estimate minimum splits sittings for every phase whenever potential.

The range of cycle length recommended by HCM is 60-120 seconds. Long cycle length exceeding 100 seconds will be recommended to accommodate an extra $10 \%$ capacity. Long cycle lengths more than when the cycle length exceeded 100 second, it will be recommended to accommodate a further more $10 \%$ capacity. The long cycle lengths more than 120 second might have negative operation sides; include inefficient use of turning lanes, long queues and blocking. Low cycle length might be better to reduce the queue which in general raise the capacity and provide a smoother traffic operation. The range of cycle times used in the optimization process was 50-200 seconds, as a default values by SYNCHRO. 
Table 2. Effectiveness Measure for Al-Ameer Intersection created by SYNCHRO 8

\begin{tabular}{|c|c|c|c|c|c|c|}
\hline 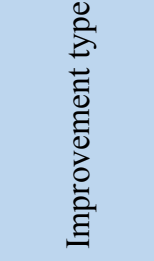 & 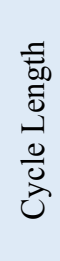 & $\stackrel{\circlearrowright}{>}$ & 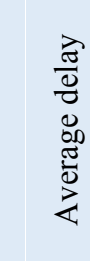 & ?ִ & 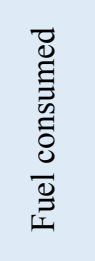 & $\begin{array}{l}\frac{8}{0} \\
\frac{0}{0} \\
0 \\
0 \\
0 \\
0 \\
0 \\
0 \\
0 \\
0\end{array}$ \\
\hline $\begin{array}{c}\text { Base } \\
\text { condition }\end{array}$ & 162 & 1.97 & 191.5 & $\mathrm{~F}$ & 942 & 1108 \\
\hline $\begin{array}{l}\text { Cycle } \\
\text { Length }\end{array}$ & 191 & 1.71 & 192.2 & $\mathrm{~F}$ & 932 & 1091 \\
\hline Splits & 162 & 1.91 & 208.8 & $\mathrm{~F}$ & 981 & 1162 \\
\hline $\begin{array}{c}\text { Phase } \\
\text { Sequences }\end{array}$ & 185 & 1.68 & 167.5 & $\mathrm{~F}$ & 816 & 947 \\
\hline $\begin{array}{l}\text { Percent } \\
\text { saving }\end{array}$ & & & $12.5 \%$ & & $13.4 \%$ & $14.5 \%$ \\
\hline
\end{tabular}

Table 2. shows that, the use of optimization functions for Al-Ameer signalized intersection has no effect, the level of service remained the same level (LOS F) each time that used the optimization functions, according to the degree of saturation $(\mathrm{V} / \mathrm{C})$, there are a few differences with this functions.

Another trial was done by changing the number of phases and the phasing sequence. The phasing order in Iraq is four-leg intersection and divided into through and left traffic with permitted right turn and yields only for pedestrians. The leading and lagging and any other protected phases are not used in this order, so the drivers are unfamiliar with this type of movements. It might cause problems with head on collisions between oncoming left turns, and also by applying this method there is no acceptable change happened.

The optimization results showed that although the cycle time increased to non-reasonable values, the intersection remains performing under oversaturated conditions, so, it is necessary to improve the performance by trying the second part of interaction strategies.

\subsection{Geometric improvements}

Since the cycle length optimization process did not improve the intersection, so it is necessary to improve the geometries of the oversaturated approaches at AlAmeer intersections by the strategies which mentioned before, and as shown in table 3.
Table 3. Effectiveness Measure of Al-Ameer Intersection that Produced SYNCHRO 8

\begin{tabular}{|c|c|c|c|c|c|c|}
\hline 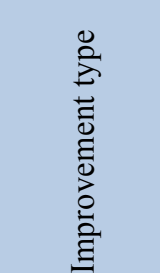 & $\begin{array}{l}5 \\
000 \\
0 \\
0 \\
0 \\
0 \\
0 \\
0\end{array}$ & $\stackrel{\bigcup}{>}$ &  & Õ &  & $\begin{array}{l}\frac{0}{0} \\
.0 \\
0 \\
D \\
0 \\
0 \\
0 \\
0 \\
0 \\
0\end{array}$ \\
\hline $\begin{array}{c}\text { Base } \\
\text { condition }\end{array}$ & 162 & 1.97 & 191.5 & $\mathrm{~F}$ & 942 & 1108 \\
\hline $\begin{array}{l}\text { Pavement } \\
\text { Remarking }\end{array}$ & 191 & 1.67 & 191.8 & $\mathrm{~F}$ & 930 & 1115 \\
\hline $\begin{array}{l}\text { Pavement } \\
\text { Widening }\end{array}$ & 171 & 1.32 & 94.5 & $\mathrm{~F}$ & 544 & 376 \\
\hline Overpass & 117 & 0.93 & 29.2 & $\mathrm{C}$ & 214 & 0 \\
\hline $\begin{array}{l}\text { Percent } \\
\text { Saving }\end{array}$ & & & $84.8 \%$ & & $77.3 \%$ & $100 \%$ \\
\hline
\end{tabular}

The first step of the geometric improvement is to apply pavement remarking. In this step consideration was given to remark the approaching lanes so that rearranging of queuing vehicles at stop line is obtained. Also, separating the movement with high traffic volume by exclusive lane is also applied. Optimization run after each improvement type is applied for the signalized intersections.

The second trail was with the pavement widening, from the measurement, it can be noticed that the number of lanes in many approaches of these intersections can be increased by decreasing the lane width (with keeping the width of lanes greater than a minimum value of 2.4 $\mathrm{m}$ according to HCM) which is actually observed to be used in field. Increasing in number of lanes will increase the saturation capacity of affected approaches, which leads to improve traffic performance measure of effectiveness (MOE) for some intersections.

The pavement remarking is applied to the intersection, but this method did not improve the measure of effectiveness (MOE), the level of service (LOS) is still with unacceptable level (LOS F), and the delay results that computed from this method for intersection is considered unsatisfied results.

By applying the second strategy the pavement widening, this method has improved the delay value at Al-Ameer intersection with $51.4 \%$ saving percent, and did not change the level of service (LOS F) were is the results shown in Table (3), using this method required enough areas and widths inside the location that intersection located in, and there is a flexibility to widen the lanes width. Al-Ameer intersection, located at restricted area.

Suggestion an overpass was the third trial, this method has applied on Al-Ameer intersection, because this 
intersection suffers from high traffic volume especially the through movement, and also, there are enough space for construction. Adopting an overpass at the east-west direction, reduced the delay to $29.2 \mathrm{sec}$ with accepted level of service (LOS C), also rearrange the cycle length to be $117 \mathrm{sec}$ as shown in Table 3 with some geometric changes and as illustrated in Figure 4 when compared with Figure 5.

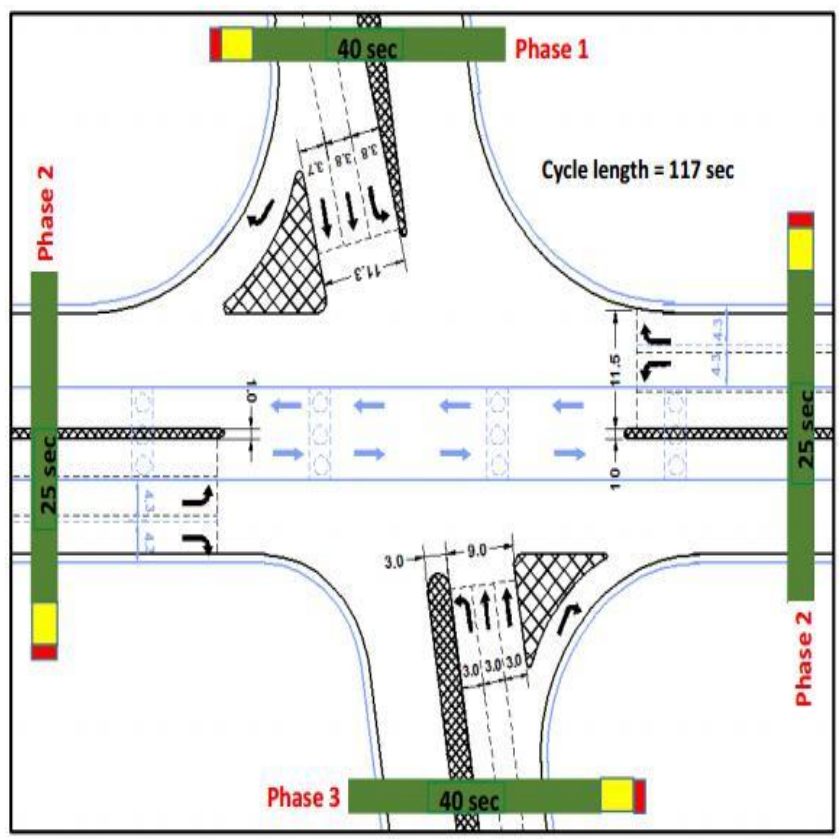

Fig. 5. Improvement proposal for Al-Ameer Interection

\section{Conclusions}

1. The local case study of Al-Ameer intersection suffer from highly congestion on four approach which lead to level of service to be at minimum requirements (level F) according to Highway Capacity Manual.

2. Using of signalization timing optimization by using SYNCHRO 8 software including splits for each phase and phase sequences shows not effective solution to improve the traffic operation performance due to high traffic volume.

3. The pavement widening strategy of geometric improvement has improved the delay value at intersection with $50.7 \%$ saving percent, and did not change the level of service (LOS F).

4. The adopting an overpass as a best strategy at the east-west direction, reduced the delay to $29.2 \mathrm{sec}$ with accepted level of service (LOS C), also rearrange the cycle length to be $117 \mathrm{sec}$.

\section{References}

1- NCC, National Council for Construction, (2003)

2- M. L. Chiguma, in Analysis of side friction impacts on Urban Roads: Case Study Dar-esSalaam. Diss .KTH (2007).

3- H. Rakha, Y. Kang and F. Dion, in Estimating Vehicle Stops at under saturated and Oversaturated Fixed-Time Signalized Intersections , (2001).

4- X. Yu and G. Sulijoadikusumo, in Assessment of Signalized Intersection Capacity in Response to Downstream Queue Spillback. (2012).

5- NACTO, in Urban Street Design Guide Overview. (2012).

6- H. Bruce and Z. Abdy, in Signalized Intersection Analysis and Design: Implications of Day-to-Day Variability in Peak Hour Volumes on Delay, Journal of Transportation Engineering 134.7 (2008): 307-318.

7- HCM, Highway Capacity Manual, (2000).

8- A. K. Jameel, in Estimating Delay Time at Palestine Street Intersection in Baghdad City Using HCM and SIDRA Models, Al-Qadisiya Journal for Engineering Sciences,4 No.1 (2011).

9- Y. Darma, M. R. Karim, J. Mohamad, and S. Abdullah, in Control Delay Variability at Signalized Intersection Based on HCM Method, 5, pp. 945 - 958 (2005).

10- NRC, "Estimation of Highway Capacity ", Rev.1, (2011)

11- Y. S. King, in Delay, Stop and Queue Estimation for Uniform and Random Traffic Arrivals at Fixed-Time Signalized Intersections (2000).

12- Trafficware, Ltd. in Traffic Signal-User Guide, Synchro Studio 8, (2011).

13- D. Husch, J. Albeck, in Traffic Signal-User Guide, Synchro Studio7, (2000). 